\title{
OC-097 CAN WE IMPROVE THE NEGATIVE PREDICTIVE VALUE OF FAECAL CALPROTECTIN FOR THE DIAGNOSIS OF IBS IN PRIMARY CARE?
}

doi:10.1136/gut.2011.239301.97

R Zayyat, ${ }^{*}$ R N Appleby, R P H Logan Department of Gastroenterology, Kings College Hospital, London, UK

Introduction Recent data has established faecal calprotectin (FC) as a highly sensitive and specific test for detecting inflammatory bowel disease (IBD) in patients referred to secondary care. ${ }^{1}$ Computer modelling has shown that the high negative predictive value (NPV) could be used to support the diagnosis of irritable bowel syndrome (IBS) in primary care, and that, after Bayesian analysis, the NPV could be increased further with higher 'cut off' of FC. ${ }^{2}$

Aim This observational cohort study aims to test this hypothesis.

Study population All patients with a borderline FC result (50-100 mg/f) from May 2005 to 2009. Patient data were cross referenced to electronic patient records, radiology (PACS), endoscopy and histology data sets using SnomedCT codes (T655260, TT67000-68930) to Oct 2010. Exclusion criteria: previous IBD diagnosis, $<16$ year at study entry or a previous FC results $>150 \mathrm{mg} / \mathrm{f}$.

Primary end point Diagnosis of IBD by standard clinical criteria during follow-up

Results From 5943 patients within the entire faecal calprotectin dataset, $433(7 \%)$ were between the values of $50-100 \mathrm{mg} / \mathrm{f}$ and met the inclusion criteria. Of 433 (62\% female, median age 48 year, range $18-90$ and $60 \%$ aged $<55$ year) diarrhoea $(56 \%)$ and alarm symptoms (11\%) were the most frequent indications for testing. The majority of patients were also consequently investigated by lower GI endoscopy ( $n=230,53 \%)$, or radiology (abdominal CT or MRI, $n=112,26 \%$ ) and $66(15 \%)$ had both tests. Endoscopic biopsies were taken in 104/230 (45\%) procedures, but which in only 10 patients confirmed a diagnosis of IBD. In all 10 patients the pretest probability of IBD was high, and in 9/10 a repeat FC had increased (median rise $35 \mathrm{mg} / \mathrm{f}$ ) in contrast to the other 52 patients with a repeat test, for who values remained the same $( \pm 15 \mathrm{mg} / \mathrm{f})$ or fell. During 
a total of 1513 patient years follow-up none of the remaining 423 patients were diagnosed with IBD.

Conclusion These observational data confirm the hypothesis that the NPV of a FC test can be safely increased by raising the cut off from 50 to $100 \mathrm{mg} / \mathrm{f}$. The results also demonstrate the potential impact of additional resource use arising from investigation of borderline FC results if the NPV is not maximised for its use in primary care. A strategy of repeat FC testing may be useful for patients with borderline results in whom the diagnosis of IBS is suspected by their GP.

Competing interests None.

Keywords diagnosis, faecal calprotectin, IBS, inflammatory bowel disease, primary care.

\section{REFERENCES}

1. van Rheenen PF, Van de Vijer E, Fidler V. Faecal calprotectin for screening of patients with suspected inflammatory bowel disease: diagnostic meta-analysis. BMJ 2010;341:c3369.

2. Whitehead SJ, Lee L, Fang Z, et al. Can faecal calprotectin reduce the demand for colonoscopy in patients with irritable bowel syndrome. Gut 2010;59(Suppl 1):A37. 